\title{
Genetic variants of VEGFR-1 gene promoter in acute myocardial infarction
}

\author{
Haihua Wang ${ }^{1}$, Shufang Zhang ${ }^{2}$, Na Wang ${ }^{3}$, Jie Zhang ${ }^{2}$, Mingkai Chen ${ }^{3}$, Xiaohui He², Yinghua Cui ${ }^{4}$, \\ Shuchao Pang ${ }^{1 *}$ and Bo Yan ${ }^{*^{*}}$ (D)
}

\begin{abstract}
Background: Coronary artery disease (CAD) including acute myocardial infarction (AMI) is a common complex disease caused by atherosclerosis. Vascular epithelial growth factor receptor-1 (VEGFR-1) stimulates angiogenesis and vascular permeability, and functions as a decoy to sequester VEGF and prevent initiation of intracellular signaling. VEGFR-1 knockout mice exhibit significantly higher mortality due to heart failure, cardiac hypertrophy, and cardiac dysfunction. An evident increase in macrophage infiltration and cardiac fibrosis are also observed after transverse aortic constriction. Therefore, VEGFR-1 gene variants may be involved in CAD. In this study, VEGFR-1 gene promoter was genetically and functionally analyzed in large cohorts of AMI patients and ethnic-matched controls.

Results: A total of 16 DNA sequence variants (DSVs) including six single-nucleotide polymorphisms (SNPs) were found in the VEGFR-1 gene promoter and 5'-untranslated region. Five novel DSVS and one SNP were only identified in AMI patients group. These DSVs and SNP significantly altered the transcriptional activity of the VEGFR-1 gene promoter in both HEK-293 and H9c2 cells $(P<0.05)$. Further electrophoretic mobility shift assay indicated that the DSVs and SNPs evidently affected the binding of transcription factors.
\end{abstract}

Conclusions: The genetic variants in VEGFR-1 gene identified in AMI patients may alter the transcriptional activity of the VEGFR-1 gene promoter and change VEGFR-1 level, contributing to AMI development.

Keywords: Acute myocardial infarction, VEGFR-1, DNA sequence variants, Genetics, Promoter

\section{Background}

Coronary artery disease (CAD) including acute myocardial infarction (AMI) is a common complex disease, which is mainly caused by atherosclerosis, an inflammatory and metabolic disease. The known risk factors for CAD include hypertension, obesity, aging, smoking, hyperlipidemia, diabetes, inflammation, and family history. A great number of common genetic variants for CAD have been identified, which only contribute to $\sim 10 \%$ of heritability [1-6]. Recently, genetic variants of low and rare frequencies have been proposed to contribute to CAD and AMI.

Proper development of the coronary vascular system is critical to deliver blood supply for myocardial growth and to ensure efficient cardiac contraction [7]. Vascular endothelial growth factor receptor-1 (VEGFR-1) is a transmembrane

\footnotetext{
*Correspondence: tonyp0720@126.com; yanbo@mail.jnmc.edu.cn

'Shandong Provincial Key Laboratory of Cardiac Disease Diagnosis and Treatment, Affiliated Hospital of Jining Medical University, Jining Medical University, 89 Guhuai Road, Jining 272029, Shandong, China

Full list of author information is available at the end of the article
}

protein, including two isoforms, a full length transmembrane VEGFR-1/Flt-1 and soluble VEGFR-1 (sVEGFR-1/ sFlt-1), which lacks the transmembrane and cytoplasmic kinase domains [8]. VEGFR-1 binds to vascular endothelial growth factor A (VEGF-A) and placental growth factor (PLGF) to stimulate angiogenesis and vascular permeability [9]. VEGFR-1 gene is homogeneously expressed in vascular epithelial cells of neonatal mouse hearts, and in coronary vessels of adult hearts [10]. sFlt-1 levels are dramatically stimulated in most cases of hypoxia [11].

Mounting evidence indicates that the function of VEGF family members extends beyond blood vessel formation. VEGFR-1 activation by VEGF-B prevents loss of cardiac mass and promotes maintenance of cardiac contractility over time [12]. The VEGFR-1 gene knockout mice have significantly higher mortality due to heart failure, and showed evident cardiac hypertrophy and cardiac dysfunction, which was accompanied by a significant increase in macrophage infiltration and cardiac fibrosis after transverse aortic constriction [13]. sVEGFR-1 regulates the

(C) The Author(s). 2019 Open Access This article is distributed under the terms of the Creative Commons Attribution 4.0 International License (http://creativecommons.org/licenses/by/4.0/), which permits unrestricted use, distribution, and 
availability of free VEGF and PLGF in peripheral circulation [14].

Recently, sVEGFR-1 has been implicated in the pathogenesis of cardiovascular disease [15-22]. Acting as a negative regulator of angiogenesis, sVEGFR-1 binds to VEGF, inhibiting the angiogenic action of VEGF in arterial endothelium [23]. Elevated levels of sVEGFR-1 have been suggested as a predictor for mortality in AMI patients [24]. Therefore, we speculated that altered expression of VEGFR-1 gene, caused by the DNA sequence variants (DSVs) with its regulatory regions, may contribute to the CAD development. In this study, we genetically analyzed the VEGFR-1 gene promoter in large cohorts of AMI patients and healthy controls.

\section{Results}

Genetic variants identified in the VEGFR-1 gene promoter and 5'-UTR

In this study population, a total of 16 DSVs were identified in the VEGFR-1gene promoter and $5^{\prime}$-untranlated region (5'-UTR), including ten novel heterozygous DSVs and six single-nucleotide polymorphisms (SNPs). Distributions and locations of the genetic variants are summarized in Table 1 and Fig. 1. Five novel heterozygous DSVs, g.28495796G > T, g.28495445G > T, g.28495341A > G, g.28495138G $>\mathrm{C}$ and g.28494900G $>\mathrm{A}$, and one SNP, g.28495805C $>\mathrm{T}$ ( $\mathrm{rs} 111458691)$, were identified in six AMI patients but in none of the controls. The DNA sequencing chromatograms of these novel DSVs were shown in Fig. 2a. Two novel heterozygous DSVs, g.28495678G $>$ A and g. $28495356 \mathrm{G}>\mathrm{A}$, were only found in two healthy controls. The DNA sequencing chromatograms of these novel DSVs were shown in Fig. 2b. The other three novel DSVs (g.28495029G $>$ T, g.28495028C $>$ A, and g. $28494895 \mathrm{G}>\mathrm{T}$ ) and five SNPs [g.28495538C $>\mathrm{T}$ ( $\mathrm{rs} 17086747)$, g. 28495 527G $>$ A (rs117031451), g.28495412C $>$ T (rs36204411), g.2 $8495135 \mathrm{G}>\mathrm{C}$ (rs17086745), and g.28494902C $>\mathrm{T}$ (rs559 27955)] were found in both AMI patients and controls with similar frequencies $(P>0.05)$. In addition, distribution

Table 1 Genetic variants in the VEGFR-1 gene promoter identified in AMI patients and controls

\begin{tabular}{|c|c|c|c|c|c|}
\hline DSVs and SNPs & Genotype & Location $^{\text {a }}$ & Controls $(n=414)$ & AMI $(n=386)$ & $P$ value \\
\hline g.28495805C>T (rs111458691) & $\mathrm{CT}$ & $-677 b p$ & 0 & 1 & - \\
\hline g.28495796G >T & GT & -668 bp & 0 & 1 & - \\
\hline g.28495678G >A & GA & $-550 \mathrm{bp}$ & 1 & 0 & - \\
\hline \multirow[t]{3}{*}{ g.28495538C>T (rs17086747) } & $\mathrm{CC}$ & -410 bp & 388 & 357 & 0.574 \\
\hline & CT & & 26 & 28 & \\
\hline & $\pi$ & & 0 & 1 & \\
\hline \multirow[t]{3}{*}{ g.28495527G>A (rs117031451) } & GG & -399 bp & 380 & 360 & 0.748 \\
\hline & GA & & 33 & 25 & \\
\hline & AA & & 1 & 1 & \\
\hline g.28495445G>T & GT & $-317 b p$ & 0 & 1 & - \\
\hline \multirow[t]{3}{*}{ g.28495412C>T (rs36204411) } & CC & $-284 \mathrm{bp}$ & 388 & 357 & 0.574 \\
\hline & CT & & 26 & 28 & \\
\hline & $\pi$ & & 0 & 1 & \\
\hline g.28495356G>A & GA & $-228 \mathrm{bp}$ & 1 & 0 & - \\
\hline g.28495341A>G & AG & $-213 b p$ & 0 & 1 & - \\
\hline g.28495138G>C & GC & $-10 \mathrm{bp}$ & 0 & 1 & - \\
\hline \multirow[t]{3}{*}{ g.28495135 G>C (rs17086745) } & GG & $-7 \mathrm{bp}$ & 388 & 357 & 0.574 \\
\hline & GC & & 26 & 28 & \\
\hline & CC & & 0 & 1 & \\
\hline g.28495029G $>T$ & GT & +99bp & 1 & 1 & 1.000 \\
\hline g.28495028C>A & CA & $+100 \mathrm{bp}$ & 1 & 1 & 1.000 \\
\hline \multirow[t]{3}{*}{ g.28494902C>T (rs55927955) } & CC & $+226 \mathrm{bp}$ & 355 & 343 & 0.162 \\
\hline & $C T$ & & 58 & 43 & \\
\hline & $\pi$ & & 1 & 0 & \\
\hline g.28494900G>A & GA & $+228 \mathrm{bp}$ & 0 & 1 & - \\
\hline g.28494895G>T & GT & $+233 b p$ & 1 & 1 & 1.000 \\
\hline
\end{tabular}

DSVs are located upstream (-) and downstream (+) to the transcription start site at 28495128 of VEGFR-1 gene (NC_000013.11). AMI acute myocardial infarction, DSV DNA sequence variant, - not applicable 


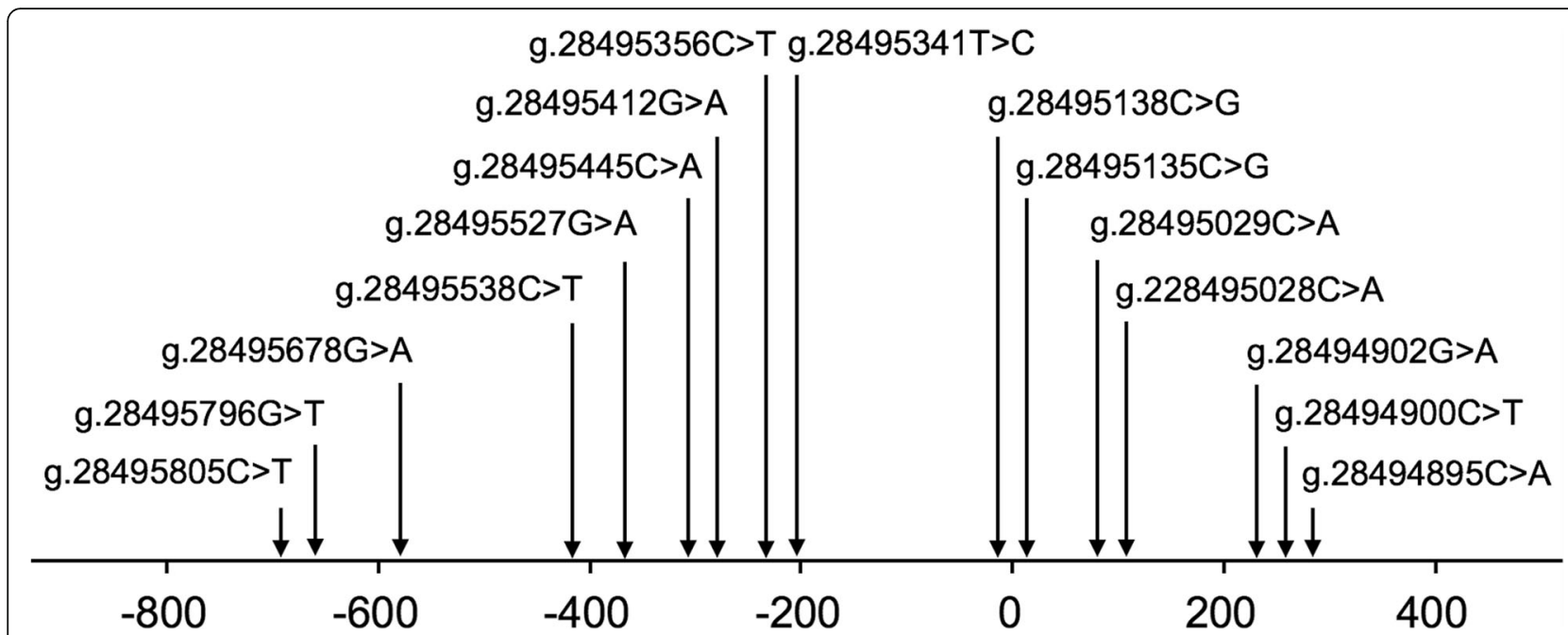

Fig. 1 Locations of the identified DSVs and SNPS within the VEGFR-1 gene promoter and 5'-UTR. The genetic variants are named according to the genomic DNA sequence of the human VEGFR-1 gene (Genbank accession number NC_000013.11). The transcription start site is at the position of 28495128 in the first exon

of all SNPs was in Hardy-Weinberg equilibrium in both groups of AMI patients and healthy controls $(P>0.05)$.

\section{Putative binding sites for transcription factors affected by genetic variants}

To determine whether the genetic variants affect putative binding sites for transcription factors, the VEGFR-1 gene promoter was analyzed with JASPAR program (http://jaspar.genereg.net/) [25].

The results showed that genetic variants found only in AMI patients or healthy controls may abolish or create binding sites for transcription factors. The analysis data were summarized in Table 2. Among these transcription factors, a Forkhead box protein O3 (FOXO3) gene SNP (rs2802292) has been reported as a protective factor against CAD mortality [26].

\section{Functional analysis of the DSVs by dual-luciferase reporter assay}

The expression vectors for luciferase reporter gene were constructed with wild-type and variant VEGFR-1 gene promoters. The expression vector for wild-type VEGFR-1 gene promoter was designated as pGL3-WT. The variant expression vectors for the DSVS in AMI patients included pGL3-28495805 T, pGL3-28495796 T, pGL3-28495445 T, pGL3-28495341G, pGL3-28495138C, and pGL3-2849 4900A. The variant expression vectors for the DSVs in healthy controls included pGL3-28495356A and pGL328495678A. Empty pGL3-basic vector was used as a negative control. Transfected human embryonic-kidney cell line (HEK-293) and rat cardiomyocyte line (H9c2) cells were collected and dual-luciferase activities were assayed. Transcriptional activity of the wild-type VEGFR-1 gene promoter was set as $100 \%$. The relative transcriptional activity of the variant VEGFR-1gene promoter was calculated compared to that of wild type VEGFR-1 gene promoter.

In HEK-293 cells, the DSVs (g.28495445G $>\mathrm{T}$, g.28495138G $>C$ ) that were identified only in AMI patients significantly decreased activity of the VEGFR1gene promoter $(P=0.004$ and $P=0.032$, respectively). In contrast, the SNP [g.28495805C $>\mathrm{T}$ ( $\mathrm{rs} 111458691)$ ] and the DSV (g.28494900G>A) identified in AMI patients significantly increased the transcriptional activity of the VEGFR-1 gene promoter $(P=0.021$ and $P=0.004$, respectively). In contrast, the DSVs (g.28495341A>G and g.28495796G $>$ T) identified in AMI patients did not affect the transcriptional activity of the VEGFR-1 gene promoter $(P>0.05)$. As expected, the DSVs found only in controls (g. 28495356G>A and g.28495678G $>$ A) did not significantly alter activity of the VEGFR-1 gene promoter $(P>0.05)$ (Fig. 3a).

To further investigate the tissue-specificity, the transcriptional activities of the variant VEGFR-1 gene promoters were examined in H9c2 cells. The DSVs (g. 28495445G>T and g. $28495138 \mathrm{G}>\mathrm{C}$ ) significantly decreased activity of the VEGFR-1 gene promoter $(P=0.009$ and $P=0.002$, respectively). The SNP [g.28495805C $>\mathrm{T}$ ( $\mathrm{rs} 111458691)$ ] and the DSV (g. 28494900G>A) significantly increased the transcriptional activity of the VEGFR-1 gene promoter $(P=$ 0.026 and $P=0.006$ respectively). Similarly, the DSVs (g. $28495356 \mathrm{G}>\mathrm{A}$ and g. $28495678 \mathrm{G}>\mathrm{A}$ ) found only in controls did not significantly alter activity of the VEGFR-1 gene promoter $(P>0.05)$ (Fig. 3b). The effects of DSVs and SNPs on VEGFR-1 gene promoter transcriptional activity were also shown in Table 2. Taken together, the DSVs and SNPs in the VEGFR-1 gene promoter identified in AMI patients affected the activity of the VEGFR-1 gene promoter in both 
A.

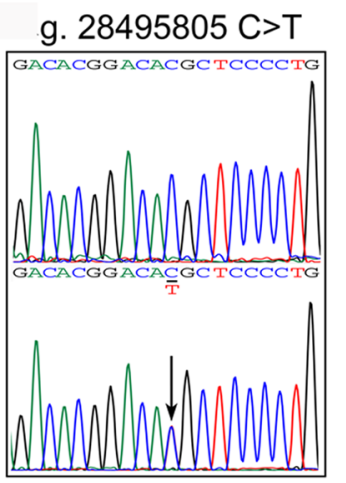

g. $28495445 \mathrm{G}>\mathrm{T}$

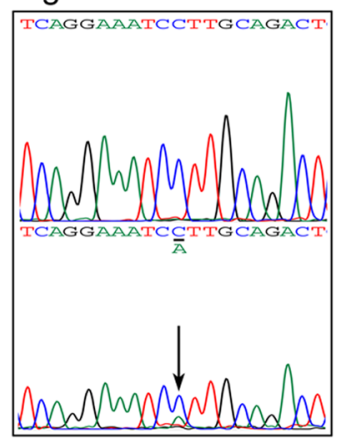

g. $28495138 \mathrm{G}>\mathrm{C}$

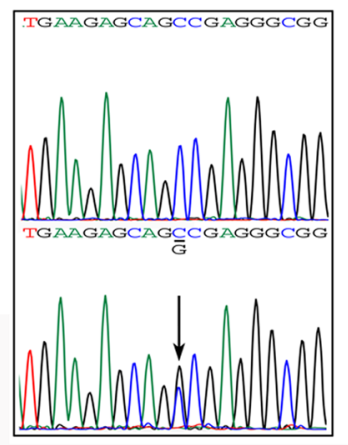

B.

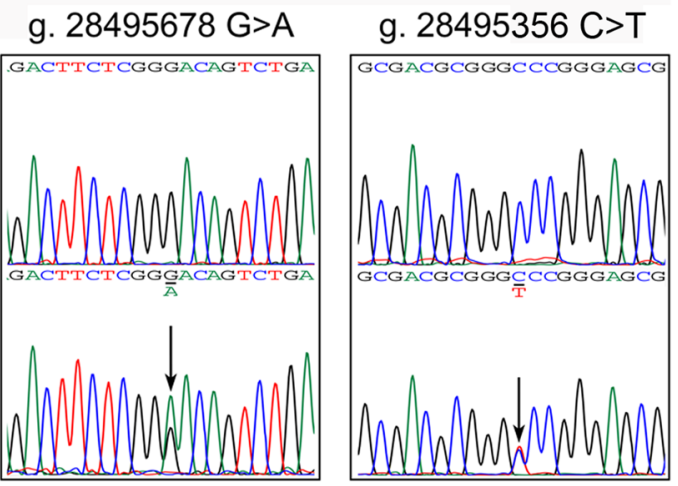

\section{g. $28495796 \mathrm{G}>\mathrm{T}$}

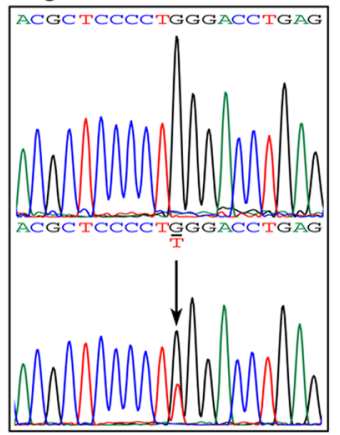

g. $28495341 A>G$

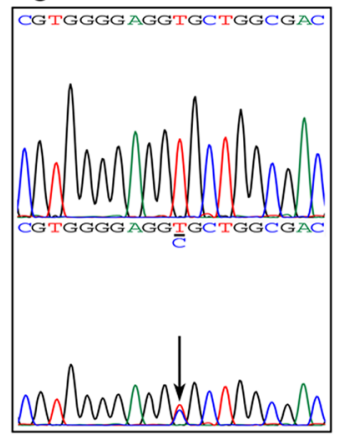

g. $28494900 \mathrm{G}>\mathrm{A}$

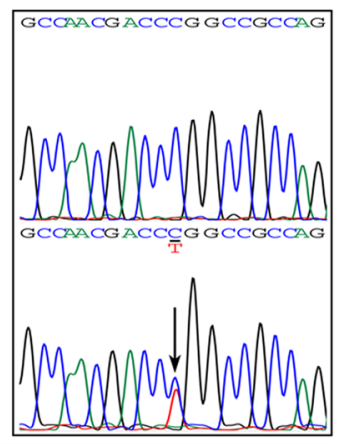

Fig. 2 Sequencing chromatograms of the DSVs and SNPs. a Sequencing chromatograms of the DSVs and SNPs only identified in AMl patients, g.28495805C > T (rs 111458691), g.28495796 G>T, g.28495445 G>T, g.28495341 A>G, g.28495138 G>C, and g.28494900 G>A. b Sequencing chromatograms of the DSVs identified only in controls, g.28495678 G>A and g.28495356G >A. Top panels show wild-type and bottom panels heterozygous sequences, which are marked with arrows

HEK-293 cells and H9c2 cells, which were non-tissue specific.

The binding sites for transcription factors affected by the DSVs

To explore whether the genetic variants affected the binding sites for transcription factors, EMSA was performed with wild-type or variant oligonucleotides (Table 3). As shown in Fig. 4, the SNP g.28495805C >T (rs111458691) weakly enhanced the binding for a transcription factor in 293 cells, which was not observed in H9c2 cells. The DSV g.28495138 G>C significantly weakened the binding of a transcription factor and enhanced the binding of another transcription factor in both 293 and H9c2 cells. The DSV g.28494900G>A created a binding of a transcription factor in both 293 and $\mathrm{H} 9 \mathrm{c} 2$ cells. However, the DSVs g.28495796G $>\mathrm{T}$, g.28495445 G>T, and g.28495341A>G did not influence the binding of transcription factors, the results of which were not shown. Therefore, these genetic variants may alter the transcription of VEGFR-1 gene through affecting the binding of transcription factors.

\section{Discussion}

To date, the VEGFR-1 gene has been implicated in human diseases. Changes in VEGFR-1 expression have been reported in human melanoma cells to stimulate cell proliferation, chemotaxis, and extracellular matrix invasion [27]. A recent study reveals that VEGFR-1 recruits $\mathrm{CD}_{133^{+}}$progenitors to inhibit local inflammation [28]. A decrease in VEGFR-1 has been associated with osteoarthritis pain [29]. sVEGFR-1 levels are increased in preeclampsia-a pathological condition of pregnancy [30]. Previous studies have shown that elevated sFlt- 1 contributes to the development of heart failure. sFlt-1 levels in plasm are directly correlated with the severity of heart failure, and also strongly associated with poor outcomes in patients with heart failure $[15,19]$. In this study, we found five novel heterozygous DSVs and one SNP in the VEGFR-1 gene promoter and 5'-UTR in six AMI patients. These DSVs and SNP significantly affected the transcriptional activity of the VEGFR-1 gene promoter by influencing the binding of transcription factors. Therefore, the genetic variants in VEGFR-1 gene may alter transcriptional activity of VEGFR-1 gene promoter 
Table 2 Predicted binding sites for transcription factors and promoter activity affected by DSVs and SNPS

\begin{tabular}{|c|c|c|c|c|}
\hline \multirow[t]{2}{*}{ DSVs/SNPS } & \multicolumn{2}{|c|}{ Binding cites for transcription factors } & \multirow{2}{*}{$\begin{array}{l}\text { Promoter } \\
\text { activity }\end{array}$} & \multirow{2}{*}{$\begin{array}{l}\text { AMl } \\
\text { CTR }\end{array}$} \\
\hline & Creating & Abolishing & & \\
\hline g.28495805C>T (rs111458691) & ELF5, NFE2L1:MafG & EGR2 & $\uparrow$ & AMl \\
\hline g.28495796G>T & - & ESRRA, NR2C2, PAX5, PPARG::RXRA, ZEB1 & No change & AMl \\
\hline g.28495678G>A & NFATC1 & TFE3 & No change & CTR \\
\hline g.28495445G>T & FOXO3, NFE2L1::MafG & NFKB1 & $\downarrow$ & AMl \\
\hline g.28495356G >A & HINFP & E2F4, TFAP2C & No change & CTR \\
\hline g.28495341A>G & EGR1, NRF1 & BRCA1, HIF1A::ARNT & No change & AMl \\
\hline g.28495138G>C & - & NHLH1, HIF1A::ARNT & $\downarrow$ & AMl \\
\hline g.28494900G $>A$ & NF1C & - & $\uparrow$ & AMl \\
\hline
\end{tabular}

and change VEGFR-1 level, contributing to AMI development as a risk factor.

Human VEGFR-1 gene has been localized to chromosome 13p12 and encodes two isoforms: a full length transmembrane VEGFR-1/Flt-1, and soluble VEGFR-1 (sVEGFR-1/sFlt-1). While expression of transmembrane VEGFR-1 and VEGF-A is increased by hypoxia, it is not clear whether sVEGFR-1 expression is downregulated or upregulated by reduced oxygen concentration [31, 32]. Ado downregulates sVEGFR-1 and upregulates VEGFR1 production to stimulate angiogenesis in the heart [33]. Mouse studies have shown that after myocardial infarction, expression of VEGFR-1 is induced in the regenerating coronary vessels at day 7 and is maintained in the newly formed coronary vessels until 30 days after myocardial infarction, recapitulating their expression kinetics during development [10].

Numerous studies have demonstrated that stimulation of angiogenesis is beneficial to ischemic and infarcted heart [34-37]. Collateral growth during ischemia requires proliferation of endothelial cells, recruitment of leukocytes and endothelial progenitor cells, and remodeling of the extracellular matrix. In the process, VEGFs play a key role via binding to VEGFR-1 and VEGFR-2 selectively [38-43]. VEGFR-1 has a primary role in embryonic angiogenesis. Mice carrying a homozygous deletion of VEGFR-1 intracellular kinase domain showed correct development of blood vessels [44]. While VEGFR-1 negatively regulates endothelial cell differentiation during development, it promotes angiogenesis under certain conditions in adult tissues, especially in ischemic tissues. Transmembrane VEGFR-1 and its selective ligands PLGF and VEGF-B have been highly involved in pathological angiogenesis $[45,46]$. Under certain circumstances upon stimulation with PLGF, a VEGFR-1specific ligand, VEGFR-1 may heterodimerize with VEGFR-2 leading to transactivation of VEGFR-2 and angiogenesis [47]. Overexpression of PLGF in transgenic mice is also reported to promote angiogenesis [48]. These findings suggest that when VEGFR-1 is paired with VEGFR-2 via a mechanism of heterodimerization, its activity positively regulates angiogenesis. Therefore, changed VEGFR-1 level may interfere with angiogenesis via above pathways.

\section{Conclusions}

In this study, we found several functional genetic variants in VEGFR-1 gene in AMI patients. These genetic variants may change VEGFR-1 level, contributing to AMI development via affecting angiogenesis. These data warrant for further investigation of the molecular mechanisms by which the genetic variants change VEGFR1 gene expression.

\section{Methods}

\section{AMI patients and healthy controls}

All AMI patients $(n=386$; age $24-86$ years, mean age 62.24 years; males 221 and females 165) were recruited from the Cardiac Care Unit in Division of Cardiology, Affiliated Hospital of Jining Medical University, Jining Medical University, Jining, Shandong, China. AMI patients were diagnosed according to clinical manifestations, abnormal electrocardiogram, and elevated levels of plasma cardiac necrosis makers. Ethnic-matched healthy controls $(n=414$; age $20-84$ years, mean age 51.33 years; males 235 and females 179) were from Physical Examination Center in the same hospital. Healthy controls with familial CAD histories were excluded from the study. This study was approved by the Human Ethics Committee of the Affiliated Hospital of Jining Medical University. Informed consents were obtained from all participates.

\section{Direct DNA sequencing}

Peripheral leukocytes were isolated venous blood $(5.0 \mathrm{ml})$. Genomic DNAs in leukocytes were extracted with DNeasy Blood and Tissue Kit (QIAGEN, Valencia, CA, USA). The putative VEGFR-1 gene promoter and $5^{\prime}$-UTR $(-1022 \mathrm{bp} \sim+284$ bp to the transcription start site) was analyzed. Two DNA fragments, $770 \mathrm{bp}$ (- $1092 \mathrm{bp} \sim-338 \mathrm{bp})$ and $788 \mathrm{bp}(-479 \mathrm{bp} \sim+308 \mathrm{bp})$, 


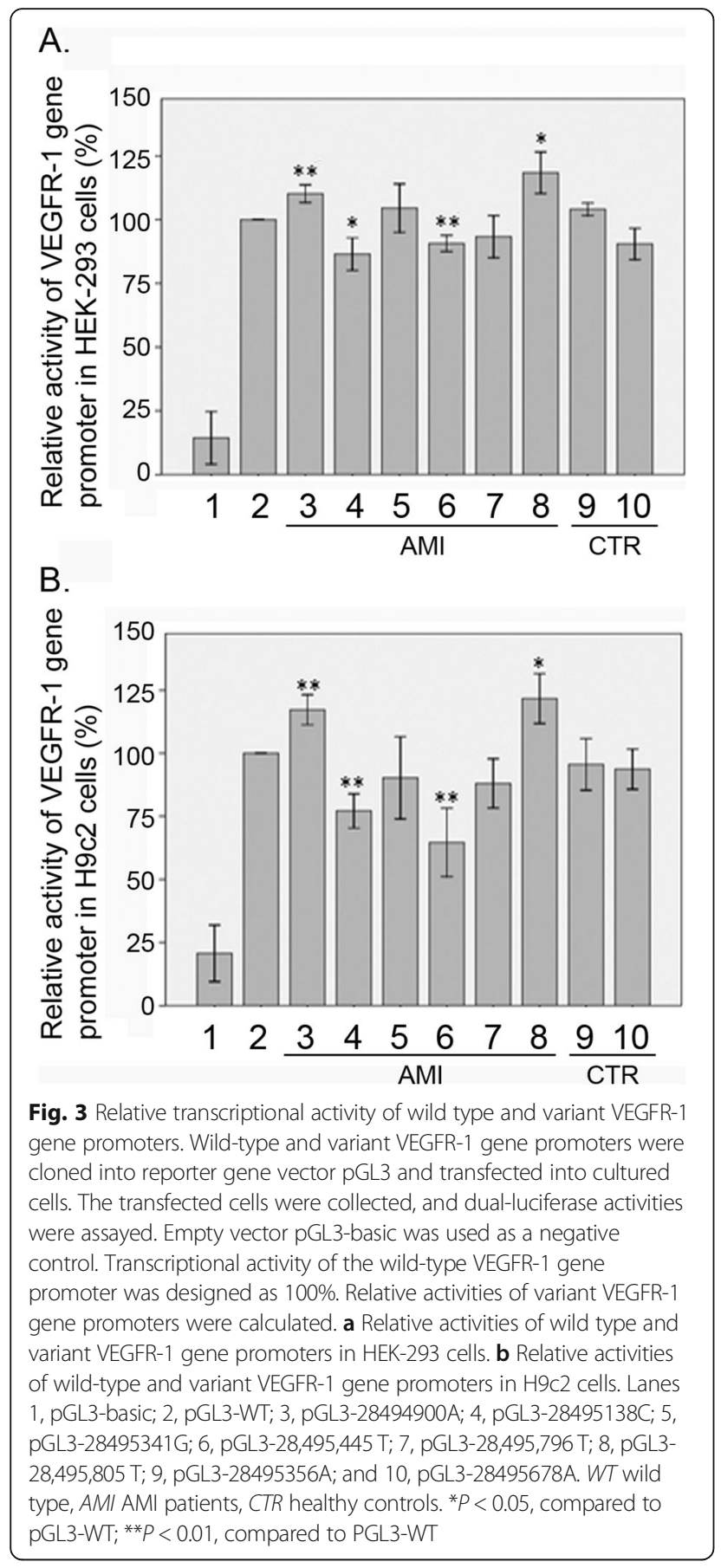

which overlappingly covered the VEGFR-1 gene promoter and 5 '-UTR, were generated by polymerase chain reaction (PCR) with Taq DNA polymerase PCR master mix (Promega Corporation, Madison, WI, USA). Genomic DNAs (100 ng) were used as PCR template. PCR primers were designed with the genomic sequence of human VEGFR-1 gene (GenBank accession number, NC_000013.11) (Table 4). PCR products were bi-directionally sequenced by Sangon Biotech Co., Ltd. (Shanghai, China) with 3730 DNA Analyzer (Applied Biosystems, Foster City, CA, USA). DNA sequences were then compared with the wild-type VEGFR1 gene promoter and 5 '-UTR using DNAMAN program (version 5.2.2; Lynnon BioSoft, Quebec, Canada), and DNA variants were identified. The DNA variants in the VEGFR-1 gene promoter were first analyzed using JASPAR program to predict their effects on binding sites for transcription factors, which were further experimentally confirmed.

\section{Functional analysis with dual-luciferase reporter assay}

Wild-type and variant VEGFR-1 gene promoter and 5'UTR (1307 bp, - 1022 bp + 284 bp) was generated by PCR and inserted into the KpnI and HindIII sites of a luciferase reporter plasmid (pGL3-basic) to generate expression vectors. PCR primers with KpnI or HindIII sites were shown in Table 4. All expression vectors were confirmed by direct DNA sequencing. Designated expression vectors were transiently transfected into human embryonic kidney cell line [HEK-293, CRL-1573; American Type Culture Collection (ATCC), Manassas, VA, USA] and rat cardiomyocyte cell line (H9c2, CRL-1446; ATCC). HEK-293 and $\mathrm{H} 9 \mathrm{c} 2$ cells were cultured at $37^{\circ} \mathrm{C}$ in $5 \% \mathrm{CO}_{2}$ humidified environment. On the day prior to transfection, the cells were seeded in 6-well plates at 40-50\% confluence. Designated expression vectors $(1.0 \mu \mathrm{g})$ and Lipofectamine ${ }^{\circ}$ (3.0 $\mu \mathrm{l}$; Thermo Fisher Scientific, Rockford, IL, USA) in $500 \mathrm{ml}$ serum-free medium were used for transfection in each well. The vector expressing Renilla luciferase (pRLTK; 25 ng, Promega Corporation) was used as an internal control for transfection efficiency. Forty-eight hours after transfection, the transfected cells were collected and luciferases activities were measured using dual-luciferase reporter assay system on a Promega Glomax 20/20 luminometer. Plasmid pGL3-basic was used as a negative control. VEGFR-1 gene promoter activity was expressed

Table 3 Double-stranded biotinylated oligonucleotides containing DSVs in AMI for EMSA

\begin{tabular}{ll}
\hline DSVs & Oligonucleotide sequences \\
\hline g.28495805C>T (rs111458691) & 5'-AAAAAGACACGGACA(C/T)GCTCCCCTGGGACC-3' \\
g.28495796G>T & 5'-CGGACACGCTCCCCT(G/T)GGACCTGAGCTGGT-3' \\
g.28495445G>T & 5'-GGAGGGAGTCTGCAA(G/T)GATTCCCTGAGCGC-3' \\
g.28495341A>G & 5'-GCCCGCGTCGCCAGC(A/G)CCTCCCCACGCGCG-3' \\
g.28495138G>C & 5'-TCGCCCCCGCCCTCG(G/C)CTGCTCTTCATCGA-3' \\
g.28494900G>A & 5'-CGGACTCTGGCGGCC(G/A)GGTCGTTGGCCGCG-3' \\
\hline
\end{tabular}




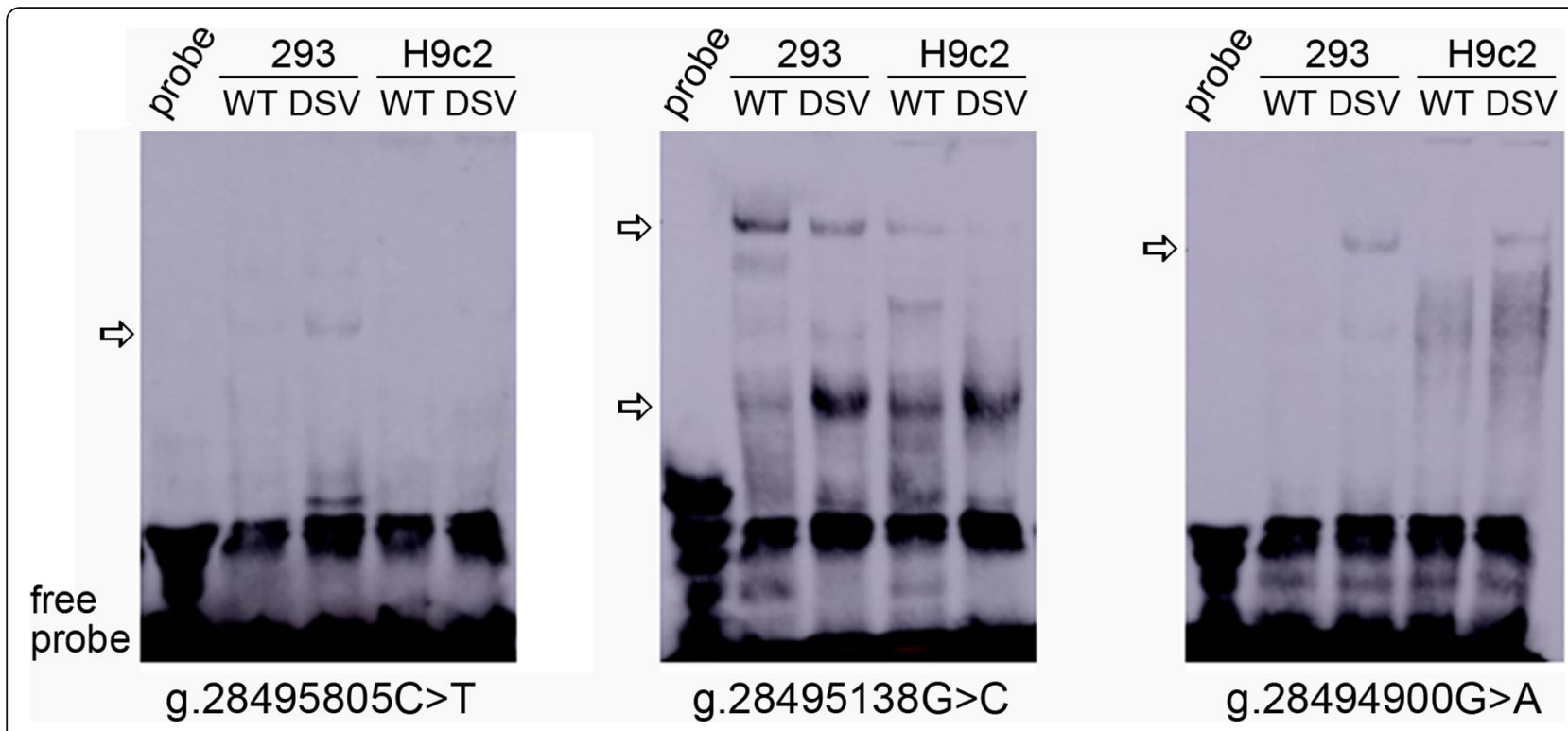

Fig. 4 EMSA of biotin-labeled oligonucleotides. Wild-type and variant oligonucleotides (30 bp) were designed and labeled with biotin for genetic variants identified in AMl patients, including g.28495805C > (rs111458691), g.28495138G>C, and g.28494900G>A. EMSA was conducted with biotinylated oligonucleotides and the nuclear extracts from HEK-293 and H9c2 cells. Free probe was marked with an arrow at the bottom. The affected binding for transcription factors was marked with an open arrow

as the ratio of luciferase activity over Renilla luciferase activity. Transcriptional activity of wild-type VEGFR-1 gene promoter was set as $100 \%$. All the experiments were repeated at least three times independently, in triplicate.

Nuclear extracts preparation and electrophoretic mobility shift assay (EMSA).

Nuclear extracts were prepared from HEK-293 and H9c2 cells using NE-PER ${ }^{\circ}$ Nuclear and Cytoplasmic Extraction Reagents (Thermo Fisher Scientific). Protein concentrations of nuclear extracts were determined with Bio-Rad protein assay reagent. EMSA was performed using the LightShift ${ }^{\circ}$ Chemiluminescent EMSA kit (Thermo Fisher Scientific) following manufacturer's protocol. Biotinylated doublestranded oligonucleotides were designed on the wild-type and variant DNA sequence. DNA-protein-binding reaction was incubated for $20 \mathrm{~min}$ at room temperature with equal amounts of biotinylated oligonucleotides $(0.2 \mathrm{pM})$ and nuclear extracts $(3.0 \mu \mathrm{g})$. The reaction mixtures were separated on a native $6 \%$ polyacrylamide gel at $100 \mathrm{~V}$ for $1 \mathrm{~h}$ at room temperature, and subsequently transferred onto a nylon membrane at $100 \mathrm{~V}$ for $30 \mathrm{~min}$. The oligonucleotides were cross-linked to the membrane using the UV Stratalinker 1800 (Stratagene, Santa Clara, CA, USA) and then detected by chemiluminescence using the LightShift ${ }^{\circ}$ Chemiluminescent EMSA kit (Thermo Fisher Scientific).

\section{Statistical analysis}

Quantitative data were represented as mean \pm SEM, and analyzed using two-way analysis of variance followed by Dunnett's test. DSV frequencies were analyzed with $x^{2}$ test using SPSS v13.0 (SPSS, Inc., Chicago, IL, USA). $P<0.05$ was considered statistically significant.

Table 4 PCR primers for the VEGFR-1 gene promoter and 5'-UTR region

\begin{tabular}{llll}
\hline PCR primers & Sequences & Location & Position \\
\hline Sequencing & & & Products \\
VEGFR-1-F1 & 5'-ACATCCCTCTGACGGGTTCCA-3' $^{\prime}$ & 28496221 & $-1092 \mathrm{bp}$ \\
VEGFR-1-R1 & 5'-GTAAGCCGGGTGGAGGGAGT-3' $^{\prime}$ & 28495471 & $-338 \mathrm{bp}$ \\
VEGFR-1-F2 & 5'-GCCTCAGTCCTCCGTGCCAAGA-3' $^{\prime}$ & 28495608 & $-479 \mathrm{bp}$ \\
VEGFR-1-R2 & 5'-ATGGTCAGCTACTGGGACACCGG-3' $^{\prime}$ & 28494821 & $+308 \mathrm{bp}$ \\
Functioning & & & $788 \mathrm{bp}$ \\
VEGFR-1-F & 5'-(Kpnl)-GCTCCGTGCAGCCAGGACGA-3' $^{\prime}$ & $28,496,151$ & $-1022 \mathrm{bp}$ \\
VEGFR-1-R & 5'-(HindIII)-GTGAGCGCGACGCGGCCT-3' $^{\prime}$ & $28,494,845$ & $+284 \mathrm{bp}$
\end{tabular}

PCR primers are designed based on the genomic DNA sequence of the VEGFR-1 gene (NC_000013.11). The transcription start is at the position of 28495128 (+ 1). $P C R$ polymerase chain reaction 


\section{Abbreviations}

5'-UTR: 5'-Untranslated region; AMl: Acute myocardial infarction; ARNT: Aryl hydrocarbon receptor nuclear translocator; BRCA1: Breast cancer 1; CAD: Coronary artery disease; CTR: Controls; DSVs: DNA sequence variantsE2F4E2F transcription factor 4; EGR1: Early growth response protein 1; EGR2: Early growth response protein 2; ELF5: E74-like factor 5;

ESRRA: Estrogen-related receptor alpha; FOXO3: Forkhead box protein O3; H9c2: Rat cardiomyocyte line; HEK-293: Human embryonic-kidney cell line; HIF1A::ARNT: Heterodimers of HIF-1A (hypoxia-inducible factor 1A) and ARNT (aryl hydrocarbon receptor nuclear translocator); HINFP: Histone H4 transcription factor; NFATC1: Nuclear factor of activated T-cells 1; NFE2L1: Nuclear factor erythroid-2-like 1; NFE2L1:MafG: Heterodimers of NFE2L1 (nuclear factor erythroid-2-like 1) and a small Maf protein; NFIC: Nuclear factor I-C; NFKB1: Nuclear factor NF-kappa-B 1; NHLH1: Nescient helix-loop-helix 1; NR2C2: Nuclear receptor subfamily 2 group C member 2; NRF1: Nuclear factor E2-related factor 1; PAX5: Paired box transcription factor 5; PLGF: Placental growth factor;

PPARG::RXRA: Heterodimers of PPARG (peroxisome proliferator-activated receptor gamma) and RXRA (retinoid X receptor-a); SNPs: Single-nucleotide polymorphisms; TFAP2C: Transcription factor AP-2 gamma;

TFE3: Transcription factor E3; VEGF: Vascular endothelial growth factor; VEGFA: Vascular endothelial growth factor A; VEGF-B: Vascular endothelial growth factor B; VEGFR-1: Vascular epithelial growth factor receptor-1; VEGFR-1/FIt1: A full length transmembrane VEGFR-1; sVEGFR-1/sFlt-1, soluble; ZEB1: Zinc finger E-box binding homeobox 1

\section{Acknowledgments}

Not applicable.

\section{Authors' contributions}

HW, SP, and BY conceived and designed the experiments. SZ, NW, JZ, MC, and $\mathrm{XH}$ performed the experiments. HW, YC, and SP analyzed the data. HW and SP wrote the manuscript. YC and BY reviewed and edited the manuscript. All authors read and approved the final manuscript.

\section{Funding}

This study was supported by the Shandong Natural Science Foundation, Shandong, China (ZR2015HL007) and Scientific Research Project of Jining Medical University (JY2015KJ035).

\section{Availability of data and materials}

All data generated or analyzed during this study are included in this published article.

\section{Ethics approval and consent to participate}

The study protocol was approved by Ethics Committee of the Affiliated Hospital of Jining Medical University. All experimental procedures complied with the Declaration of Helsinki (2003). Written informed consent was obtained from all participates in the present study.

\section{Consent for publication}

Not applicable.

\section{Competing interests}

The authors declare that they have no competing interests.

\section{Author details}

'Shandong Provincial Key Laboratory of Cardiac Disease Diagnosis and Treatment, Affiliated Hospital of Jining Medical University, Jining Medical University, 89 Guhuai Road, Jining 272029, Shandong, China. ${ }^{2}$ Division of Cardiology, Affiliated Hospital of Jining Medical University, Jining Medical University, Jining, Shandong, China. ${ }^{3}$ Division of Laboratory Medicine, Affiliated Hospital of Jining Medical University, Jining Medical University, Jining, Shandong, China. ${ }^{4}$ Cardiac Care Unit, Affiliated Hospital of Jining Medical Uniiversity, Jining Medical University, Jining, Shandong, China.
Received: 9 April 2019 Accepted: 9 October 2019

Published online: 19 November 2019

\section{References}

1. Assimes $T L$, Roberts R. Genetics: implications for prevention and management of coronary artery disease. J Am Coll Cardiol. 2016;68(25): 2797-818

2. Bjorkegren JLM, Kovacic JC, Dudley JT, Schadt EE. Genome-wide significant loci: how important are they? Systems genetics to understand heritability of coronary artery disease and other common complex disorders. J Am Coll Cardiol. 2015;65(8):830-45

3. Deloukas P, Kanoni S, Willenborg C, Farrall M, Assimes TL, Thompson JR, et al. Large-scale association analysis identifies new risk loci for coronary artery disease. Nat Genet. 2013;45(1):25-33.

4. McPherson R, Tybjaerg-Hansen A. Genetics of coronary artery disease. Circ Res. 2016;118(4):564-78

5. Ozaki K, Tanaka T. Molecular genetics of coronary artery disease. J Hum Genet. 2016;61(1):71-7.

6. Roberts R. Genetics of coronary artery disease. Circ Res. 2014;114(12):1890-903.

7. Giordano FJ, Gerber HP, Williams SP, VanBruggen N, Bunting S, Ruiz-Lozano $P$, et al. A cardiac myocyte vascular endothelial growth factor paracrine pathway is required to maintain cardiac function. Proc Natl Acad Sci U S A. 2001:98(10):5780-5.

8. Kendall RL, Thomas KA. Inhibition of vascular endothelial cell growth factor activity by an endogenously encoded soluble receptor. Proc Natl Acad Sci U S A. 1993;90(22):10705-9.

9. Carmeliet P, Moons L, Luttun A, Vincenti V, Compernolle V, De Mol M, et al. Synergism between vascular endothelial growth factor and placental growth factor contributes to angiogenesis and plasma extravasation in pathological conditions. Nat Med. 2001;7(5):575-83.

10. Kurotsu S, Osakabe R, Isomi M, Tamura F, Sadahiro T, Muraoka N, et al. Distinct expression patterns of Flk1 and Flt 1 in the coronary vascular system during development and after myocardial infarction. Biochem Biophys Res Commun. 2018;495(1):884-91.

11. Rajakumar A, Powers RW, Hubel CA, Shibata E, von Versen-Hoynck F, Plymire D, et al. Novel soluble Flt-1 isoforms in plasma and cultured placental explants from normotensive pregnant and preeclamptic women. Placenta. 2009:30(1):25-34

12. Zentilin L, Puligadda U, Lionetti V, Zacchigna S, Collesi C, Pattarini L, et al. Cardiomyocyte VEGFR-1 activation by VEGF-B induces compensatory hypertrophy and preserves cardiac function after myocardial infarction. FASEB J. 2010;24(5):1467-78.

13. Seno A, Takeda Y, Matsui M, Okuda A, Nakano T, Nakada Y, et al. Suppressed production of soluble Fms-like tyrosine Kinase-1 contributes to myocardial remodeling and heart failure. Hypertension. 2016;68(3):678-87.

14. Shibuya M. Vascular endothelial growth factor receptor-1 (VEGFR-1/FIt-1): a dual regulator for angiogenesis. Angiogenesis. 2006:9(4):225-30. discussion 31

15. Di Marco GS, Kentrup D, Reuter S, Mayer AB, Golle L, Tiemann K, et al. Soluble Flt-1 links microvascular disease with heart failure in CKD. Basic Res Cardiol. 2015;110(3):30

16. Hammadah M, Georgiopoulou W, Kalogeropoulos AP, Weber M, Wang X, Samara MA, et al. Elevated soluble Fms-like tyrosine Kinase-1 and placentallike growth factor levels are associated with development and mortality risk in heart failure. Circ Heart Fail. 2016;9(1):e002115.

17. Kaza E, Ablasser K, Poutias D, Griffiths ER, Saad FA, Hofstaetter JG, et al. Upregulation of soluble vascular endothelial growth factor receptor-1 prevents angiogenesis in hypertrophied myocardium. Cardiovasc Res. 2011:89(2):410-8.

18. Kodama Y, Kitta Y, Nakamura T, Takano H, Umetani K, Fujioka D, et al. Atorvastatin increases plasma soluble Fms-like tyrosine kinase-1 and decreases vascular endothelial growth factor and placental growth factor in association with improvement of ventricular function in acute myocardial infarction. J Am Coll Cardiol. 2006:48(1):43-50.

19. Ky B, French B, Ruparel K, Sweitzer NK, Fang JC, Levy WC, et al. The vascular marker soluble fms-like tyrosine kinase 1 is associated with disease severity and adverse outcomes in chronic heart failure. J Am Coll Cardiol. 2011:58(4):386-94.

20. Matsui M, Takeda Y, Uemura S, Matsumoto T, Seno A, Onoue K, et al. Suppressed soluble Fms-like tyrosine kinase-1 production aggravates atherosclerosis in chronic kidney disease. Kidney Int. 2014;85(2):393-403. 
21. Onoue K, Uemura S, Takeda Y, Somekawa S, Iwama H, Imagawa K, et al. Reduction of circulating soluble fms-like tyrosine kinase-1 plays a significant role in renal dysfunction-associated aggravation of atherosclerosis. Circulation. 2009;120(24):2470-7.

22. Wang Y, Zhou Y, He L, Hong K, Su H, Wu Y, et al. Gene delivery of soluble vascular endothelial growth factor receptor-1 (sFlt-1) inhibits intra-plaque angiogenesis and suppresses development of atherosclerotic plaque. Clin Exp Med. 2011;11(2):113-21.

23. Ahmad S, Ahmed A. Elevated placental soluble vascular endothelial growth factor receptor-1 inhibits angiogenesis in preeclampsia. Circ Res. 2004;95(9):884-91.

24. Hochholzer W, Reichlin T, Stelzig C, Hochholzer K, Meissner J, Breidthardt T, et al. Impact of soluble fms-like tyrosine kinase-1 and placental growth factor serum levels for risk stratification and early diagnosis in patients with suspected acute myocardial infarction. Eur Heart J. 2011;32(3):326-35.

25. Sandelin A, Alkema W, Engström P, Wasserman WW, Lenhard B. JASPAR: an open-access database for eukaryotic transcription factor binding profiles. Nucleic Acids Res. 2004;32(Database issue):D91-4.

26. Willcox BJ, Morris BJ, Tranah GJ, Chen R, Masaki KH, He Q, et al. Longevityassociated $\mathrm{FOXO} 3$ genotype and its impact on coronary artery disease mortality in Japanese, whites, and blacks: a prospective study of three American populations. J Gerontol A Biol Sci Med Sci. 2017;72(5):724-8

27. Graziani G, Ruffini F, Tentori L, Scimeca M, Dorio AS, Atzori MG, et al. Antitumor activity of a novel anti-vascular endothelial growth factor receptor-1 monoclonal antibody that does not interfere with ligand binding. Oncotarget. 2016;7(45):72868-85.

28. Qiu Y, Chen C, Zhang J, Chen M, Gong H, Gong L, et al. VEGF attenuates lung injury by inducing homing of CD133(+) progenitors via VEGFR1. Biochem Biophys Res Commun. 2019;511(3):650-7.

29. Das V, KC R, Li X, OS I, van Wijnen AJ, Kroin JS, et al. Blockade of vascular endothelial growth factor receptor-1 (Flt-1), reveals a novel analgesic for osteoarthritis-induced joint pain. Gene Rep. 2018;11:94-100.

30. Deepak V, Sahu MB, Yu J, Jones JW, Kane MA, Taylor RN, et al. Retinoic acid is a negative regulator of sFLT1 expression in decidual stromal cells, and its levels are reduced in preeclamptic decidua. Hypertension. 2019; https://doi. org/10.1161/Hypertensionaha11812564.

31. Gerber HP, Condorelli F, Park J, Ferrara N. Differential transcriptional regulation of the two vascular endothelial growth factor receptor genes. Flt1, but not Flk-1/KDR, is up-regulated by hypoxia. J Biol Chem. 1997;272(38): 23659-67.

32. Shima DT, Deutsch U, D'Amore PA. Hypoxic induction of vascular endothelial growth factor (VEGF) in human epithelial cells is mediated by increases in mRNA stability. FEBS Lett. 1995;370(3):203-8.

33. Leonard F, Devaux $Y$, Vausort M, Ernens I, Rolland-Turner M, Wagner DR. Adenosine modifies the balance between membrane and soluble forms of Flt-1. J Leukoc Biol. 2011;90(1):199-204.

34. Ahn A, Frishman WH, Gutwein A, Passeri J, Nelson M. Therapeutic angiogenesis: a new treatment approach for ischemic heart disease-part II. Cardiol Rev. 2008;16(5):219-29.

35. Boodhwani M, Sodha NR, Laham RJ, Sellke FW. The future of therapeutic myocardial angiogenesis. Shock. 2006;26(4):332-41.

36. Nelson MA, Passeri J, Frishman WH. Therapeutic angiogenesis: a new treatment modality for ischemic heart disease. Heart Dis. 2000;2(4):314-25.

37. Pandya NM, Dhalla NS, Santani DD. Angiogenesis - a new target for future therapy. Vasc Pharmacol. 2006:44(5):265-74.

38. Clayton JA, Chalothorn D, Faber JE. Vascular endothelial growth factor-a specifies formation of native collaterals and regulates collateral growth in ischemia. Circ Res. 2008;103(9):1027-36.

39. Kocaman SA, Arslan U, Tavil Y, Okuyan H, Abaci A, Cengel A. Increased circulating monocyte count is related to good collateral development in coronary artery disease. Atherosclerosis. 2008;197(2):753-6.

40. Lahteenvuo JE, Lahteenvuo MT, Kivela A, Rosenlew C, Falkevall A, Klar J, et al. Vascular endothelial growth factor-B induces myocardium-specific angiogenesis and arteriogenesis via vascular endothelial growth factor receptor-1- and neuropilin receptor-1-dependent mechanisms. Circulation. 2009;119(6):845-56.

41. Matsudaira K, Maeda K, Okumura N, Yoshikawa D, Morita Y, Mitsuhashi H, et al. Impact of low levels of vascular endothelial growth factor after myocardial infarction on 6-month clinical outcome. Results from the Nagoya Acute Myocardial Infarction Study. Circ J. 2012;76(6):1509-16.
42. Taniguchi T, Sawada T. Significance of measuring plasma vascular endothelial growth factor in patients with acute myocardial infarction. Circ J. 2012;76(6):1331-2

43. Tokgozoglu L, Yorgun $\mathrm{H}$, Gurses KM, Canpolat U, Ates AH, Tulumen $\mathrm{E}_{\text {, et }}$ al. The association between circulating endothelial progenitor cells and coronary collateral formation. Atherosclerosis. 2011;219(2):851-4.

44. Hiratsuka S, Minowa O, Kuno J, Noda T, Shibuya M. Flt-1 lacking the tyrosine kinase domain is sufficient for normal development and angiogenesis in mice. Proc Natl Acad Sci U S A. 1998;95(16):9349-54.

45. Bry M, Kivela R, Leppanen VM, Alitalo K. Vascular endothelial growth factor-B in physiology and disease. Physiol Rev. 2014;94(3):779-94.

46. Dewerchin M, Carmeliet P. PIGF: a multitasking cytokine with diseaserestricted activity. Cold Spring Harb Perspect Med. 2012;2(8).

47. Autiero M, Waltenberger J, Communi D, Kranz A, Moons L, Lambrechts D, et al. Role of PIGF in the intra- and intermolecular cross talk between the VEGF receptors Flt1 and Flk1. Nat Med. 2003;9(7):936-43.

48. Odorisio T, Schietroma C, Zaccaria ML, Cianfarani F, Tiveron C, Tatangelo L, et al. Mice overexpressing placenta growth factor exhibit increased vascularization and vessel permeability. J Cell Sci. 2002;115(Pt 12):2559-67.

\section{Publisher's Note}

Springer Nature remains neutral with regard to jurisdictional claims in published maps and institutional affiliations.

\section{Ready to submit your research? Choose BMC and benefit from:}

- fast, convenient online submission

- thorough peer review by experienced researchers in your field

- rapid publication on acceptance

- support for research data, including large and complex data types

- gold Open Access which fosters wider collaboration and increased citations

- maximum visibility for your research: over $100 \mathrm{M}$ website views per year

At BMC, research is always in progress.

Learn more biomedcentral.com/submissions 